\title{
Article \\ Genome-Wide and Comprehensive Analysis of the Multiple Stress-Related CAF1 (CCR4-Associated Factor 1) Family and Its Expression in Poplar
}

\author{
Pu Wang, Lingling Li, Hui Wei, Weibo Sun, Peijun Zhou, Sheng Zhu, Dawei Li (D) and Qiang Zhuge *(D)
}

check for updates

Citation: Wang, P.; Li, L.; Wei, H.; Sun, W.; Zhou, P.; Zhu, S.; Li, D.; Zhuge, Q. Genome-Wide and Comprehensive Analysis of the Multiple Stress-Related CAF1 (CCR4-Associated Factor 1) Family and Its Expression in Poplar. Plants 2021, 10, 981. https://doi.org/ $10.3390 /$ plants10050981

Academic Editor: Vladimir Kuznetsov

Received: 10 April 2021

Accepted: 12 May 2021

Published: 14 May 2021

Publisher's Note: MDPI stays neutral with regard to jurisdictional claims in published maps and institutional affiliations.

Copyright: (c) 2021 by the authors. Licensee MDPI, Basel, Switzerland. This article is an open access article distributed under the terms and conditions of the Creative Commons Attribution (CC BY) license (https:// creativecommons.org/licenses/by/ $4.0 /)$.
Co-Innovation Center for Sustainable Forestry in Southern China, Key Laboratory of Forest Genetics \& Biotechnology, Ministry of Education, College of Biology and the Environment, Nanjing Forestry University, Nanjing 210037, China; wangpu@njfu.edu.cn (P.W.); linglingli@njfu.edu.cn (L.L.); HW@njfu.edu.cn (H.W.); czswb@njfu.edu.cn (W.S.); pjzhou@njfu.edu.cn (P.Z.); zhusheng303@163.com (S.Z.); dwli@njfu.edu.cn (D.L.)

* Correspondence: qzhuge@njfu.edu.cn; Fax: +86-25-85428701

\begin{abstract}
Poplar is one of the most widely used tree in afforestation projects. However, it is susceptible to abiotic and biotic stress. CCR4-associated factor 1 (CAF1) is a major member of CCR4-NOT, and it is mainly involved in transcriptional regulation and mRNA degradation in eukaryotes. However, there are no studies on the molecular phylogeny and expression of the CAF1 gene in poplar. In this study, a total of 19 PtCAF1 genes were identified in the Populus trichocarpa genome. Phylogenetic analysis of the PtCAF1 gene family was performed with two closely related species (Arabidopsis thaliana and Oryza sativa) to investigate the evolution of the PtCAF1 gene. The tissue expression of the PtCAF1 gene showed that 19 PtCAF1 genes were present in different tissues of poplar. Additionally, the analysis of the expression of the PtCAF1 gene showed that the CAF1 family was up-regulated to various degrees under biotic and abiotic stresses and participated in the poplar stress response. The results of our study provide a deeper understanding of the structure and function of the PtCAF1 gene and may contribute to our understanding of the molecular basis of stress tolerance in poplar.
\end{abstract}

Keywords: CCR4 associated factor 1; poplar; gene expression; multiple stresses

\section{Introduction}

Gene expression is an important factor in plant growth and environmental communication. The steady-state level of intracellular mRNA is determined by the rate of transcription and post-transcriptional regulation of mRNA decay [1,2]. Genome-wide studies show that gene expression undergoes extensive reprogramming under environmental stimulation, and the change in the mRNA degradation rate provides a mechanism for rapidly changing mRNA abundance [3,4]. Degradation of poly(A) tail is a key step in reducing and controlling the gene expression of messenger RNA (mRNA) [5-7]. The carbon decomposing metabolite inhibitor 4 (CCR4)-associated factor 1 in CCR4-NOT deaminase complex plays an important role in the process of mRNA deamination in most eukaryotes [8,9]. CCR4NOT is a multisubunit protein complex, which is highly conserved in eukaryotes. It is mainly involved in transcription regulation, mRNA degradation, histone modification, and other important physiological processes [10-12]. CAF1 (CCR4-associated factor 1) is also called CCR4-NOT transcription complex subunit 7 (CNOT7), which is highly conserved and mainly involved in transcriptional regulation and mRNA degradation [13-15]. CAF1 is a highly conserved exonuclease in evolution. There is only a single gene in yeast, Caenorhabditis Elegans, and Drosophilid species. CAF1 belongs to the DEDD family and is one of the major degradation enzymes involved in mRNA degradation [16-18]. Although CAF1 has been widely studied in yeast and animals, its role has rarely been studied in plants.

Plants face a variety of abiotic and biotic stresses, such as plant pathogens, drought, cold, hot, and salt stress, in the process of growth, which are detrimental to their growth. 
However, plants have their own regulatory pathways with which to deal with various stresses [19]. Various studies have shown that CAF1 plays an important role in plant growth, stress response, and resistance to microbial pathogens. CAF1 is very important for the growth of yeast and animal cells, but they generally only have one or two CAF1 homologous genes, unlike the higher plants, which contain the entire CAF1 gene family [19-22]. Until now, 18 CAF1 genes homologues have been identified in Oryza sativa and 11 CAF1 genes in Arabidopsis thaliana [23]. Studies on the function of the CAF1 gene in A. thaliana and O. sativa have been published. The results show that the CAF1 gene plays an important role in plant growth and responses to biotic and abiotic stresses. Various studies have demonstrated that the overexpression of the pepper gene $C a C A F 1$ in tomato significantly promotes the growth of tomato and resistance against the oomycete pathogen Phytophthora infestans [24]. Moreover, Citrus sinensis CAF1 (CsCAF1) is involved in the stress process of Xanthomonas citri. In addition, CsCAF1 can interact with PTHA4, which is the main effective factor of $X$. citri. PTHA4 can stabilize the expression of CsLOB1 by inhibiting the deadenylase activity of CsCAF1 and can make citrus resistant to canker [25]. In $A$. thaliana, the single and double mutants of $A t C A F 1 A$ and $A t C A F 1 B$ were shown to decrease the expression of PATHOGENESIS-RELATED 1 (PR1) and PATHOGENESIS-RELATED 2 (PR2), and plants become more susceptible to Pseudomonas syringae po tomato DC3000 (PST DC3000) infection; while plants overexpressing AtCAF1A showed high expression of PR1 and PR2 and exhibited enhanced resistance to the same pathogen [26]. The CAF1 gene also plays an important role in abiotic stress response. In A. thaliana, abscisic acid (ABA), salicylic acid (SA), jasmonic acid (JA), methyl jasmonate (MeJA), and other hormones, as well as various external stresses, such as cold and wounds, can induce the rapid expression of the CAF1 gene. Furthermore, using poly(A) tail length (PAT) analysis, it was confirmed that the $C A F 1$ gene has $3^{\prime}-5^{\prime}$ amino acids. Exonuclease activity can regulate the degradation of mRNA in vivo. Stress tolerance experiments showed that $A t C A F 1 A$ and $A t C A F 1 B$ were involved in mediating abiotic stress responses [27,28]. In O. sativa, OsCAF1B plays an important role in the growth and development of $O$. sativa seedlings at low temperature. Moreover, it plays an important role in O. sativa germination and seedling growth [29,30]. In Dunaliella salina, the promoters of DsCAF1 contain many elements that are sensitive to abiotic stress. DsCAF1 was shown to be highly expressed in a high salt environment. When $D$. salina cells were subjected to stress shock, the expression of DsCAF1 demonstrated a two-stage reaction. The mRNA level of DsCAF1 increased 2-4-fold immediately after hypertonic, heat, or ultraviolet treatment, then increased 10-fold. In addition, it rapidly increased approximately 3 -fold after hypotonic or cold shock, then decreased suddenly. The different expression patterns of DsCAF1 indicate that DsCAF1 is a nuclease in response to stress, which can regulate the mRNA expression of related genes [31].

Poplar (Populus spp.) is one of the most widely distributed and adaptable tree species in the world. Because of the value of poplar in the wood industry and its advantages for afforestation, it is important both economically and ecologically and plays an important role in the global ecosystem [32,33]. At present, the molecular mechanism of stress tolerance in poplar has not been fully elucidated. As a result of the availability of the Populus trichocarpa genome sequence, poplar has been used as a model species for the study of the perennial plant genome. At present, it is very important to improve poplar varieties by means of genetic engineering. However, there are no published papers concerning the CAF1 gene in poplar. For this reason, it is necessary to study the CAF1 gene family in poplar. In this study, we characterized the CAF1 gene family in P. trichocarpa and analyzed its distribution, phylogenetic relationships, gene structure, and evolutionary characteristics. Moreover, we analyzed its expression in different tissues and its response to multiple environmental stresses. Our results provide insights that may of use for poplar breeding programs. 


\section{Results}

\subsection{Identification of the CAF1 Proteins in P. trichocarpa}

To identify the CAF1 genes in the genome of P. trichocarpa, we used the reported CAF1 in A. thaliana and O. sativa as queries in Phytozome v12.1. All possible PtCAF1 genes were excavated from the P. trichocarpa genome using the BLAST and HMMER search methods. After removing the redundant genes, 19 potential PtCAF1 genes were screened out in the P. trichocarpa genome and named PtCAF1A-PtCAF1S based on their chromosomal location. The 19 potential PtCAF1 genes were then identified and analyzed. The gene characteristics included the coding sequence length (CDS), protein amino acid quantity, protein molecular weight (MW), isoelectric point (PI), and subcellular localization (Table 1). The results showed that the 19 PtCAF1 gene code sequence lengths ranged from $648 \mathrm{bp}$ (PtCAF1B) to $1881 \mathrm{bp}$ (PtCAF1O), and the 19 PtCAF1 genes transcription factor proteins ranged from $216 \mathrm{AA}$ to $627 \mathrm{AA}$; the protein sequence encoded by PtCAF1O was the longest (627 AA), and the protein sequence encoded by PtCAF1B was the shortest (216 AA). The MW of the proteins ranged from $24.78 \mathrm{KDa}$ to $70.23 \mathrm{KDa}$, and the PI ranged from 4.41 (PtCAF1Q) to 9.91 (PtCAF1C). Understanding the subcellular localization of PtCAF1 is very important for studying and evaluating gene function. The results showed that among the 19 PtCAF1 genes, PtCAF1A, PtCAF1B, PtCAF1C, PtCAF1D, PtCAF1F, PtCAF1I, PtCAF1J, PtCAF1L, PtCAF1M, PtCAF1P, PtCAF1Q, PtCAF1R, and PtCAF1S are located in cytosol. PCAF1E and PtCAF1G are located in the nucleus. PtCAF1H, PtCAF1K, PtCAF1N, and PtCAF1O are located in the chloroplast. Most of the PtCAF1 gene localization is in cytosol, which is consistent with its function.

Table 1. List of 19 PtCAF1 genes and their basic characterizations.

\begin{tabular}{cccccccc}
\hline Name & Phytozome Gene ID & Ensembl Gene ID & CDS & AA & MW (kDa) & pI & Subcellular Location \\
\hline PtCAF1A & Potri.001G038500.1 & PNT52564 & 876 & 292 & 33.40 & 6.86 & cytosol \\
PtCAF1B & Potri.001G039000.1 & PNT52569 & 648 & 216 & 24.78 & 8.47 & cytosol \\
PtCAF1C & Potri.001G040400.1 & PNT52586 & 789 & 263 & 30.25 & 9.91 & cytosol \\
PtCAF1D & Potri.001G046700.1 & PNT52695 & 825 & 275 & 31.30 & 4.56 & cytosol \\
PtCAF1E & Potri.001G368400.1 & PNT58721 & 1425 & 475 & 53.50 & 7.85 & nucleus \\
PtCAF1F & Potri.003G181100.1 & PNT46250 & 825 & 275 & 31.22 & 4.49 & cytosol \\
PtCAF1G & Potri.003G186300.1 & PNT46340 & 915 & 305 & 34.53 & 5.21 & nucleus \\
PtCAF1H & Potri.004G048800.1 & PNT39610 & 828 & 276 & 31.98 & 6.37 & chloroplast \\
PtCAF1I & Potri.004G200400.1 & PNT42179 & 651 & 217 & 25.12 & 4.62 & cytosol \\
PtCAF1J & Potri.006G187200.1 & PNT32402 & 810 & 270 & 30.31 & 5.34 & cytosol \\
PtCAF1K & Potri.006G205600.1 & PNT32745 & 888 & 296 & 33.70 & 5.96 & chloroplast \\
PtCAF1L & Potri.006G262500.1 & PNT33885 & 834 & 278 & 31.49 & 4.46 & cytosol \\
PtCAF1M & Potri.009G161500.1 & PNT21675 & 834 & 278 & 31.71 & 5.08 & cytosol \\
PtCAF1N & Potri.014G018500.1 & PNT02530 & 1815 & 605 & 67.72 & 7.27 & chloroplast \\
PtCAF1O & Potri.014G177400.1 & PNT05527 & 1881 & 627 & 70.23 & 6.12 & chloroplast \\
PtCAF1P & Potri.016G073000.1 & PNS98364 & 891 & 297 & 33.43 & 5.8 & cytosol \\
PtCAF1Q & Potri.018G020900.1 & PNS92197 & 834 & 278 & 31.42 & 4.41 & cytosol \\
PtCAF1R & Potri.018G036100.1 & PNS92495 & 918 & 306 & 33.97 & 9.1 & cytosol \\
PtCAF1S & Potri.018G038700.1 & PNS92540 & 909 & 303 & 33.75 & 8.8 & cytosol \\
\hline
\end{tabular}

Note: CDS is coding sequence length, AA is protein amino acid quantity, MW is protein molecular weight, PI is isoelectric point.

\subsection{Multiple Sequence Alignment and Phylogenetic Analysis}

To explore the phylogenetic relationship of PtCAF1 proteins in P. trichocarpa, we constructed a phylogenetic tree using the neighbor-joining (NJ) method based on multiple sequence alignments of $O$. sativa, A. thaliana, Eucalyptus grandis, Elaeis guineensis and O. sativa, Capsicum annuum, Citrus sinensis, Nicotiana tabacum, and P. trichocarpa CAF1 protein sequences. According to previous studies on the CAF1 gene family in A. thaliana, we divided the phylogenetic tree into three groups. The 19 candidate PtCAF1 genes were divided into three groups: Group I, Group II, and Group III (Figure 1). To further evaluate the similar regions and conserved sites of the PtCAF1 genes and clarify their 
evolutionary relationship among species, we used ClustalX 2.1 for multiple sequence alignment. The amino acid sequence analysis showed that the homologues of $A$. thaliana, O. sativa, C. annuum, C. sinensis, N. tabacum, yeast (SpCAF1), human (HsCNOT7), mouse, Zea mays (ZmCAF1), and P. trichocarpa (group I) were well conserved in the RNase D domain, with three conserved motifs and four important nuclease activity catalytic residues DEDD. In addition, these CAF1 homologues contain the fifth conserved amino acid residue histidine (Supplementary Materials, Figure S1).

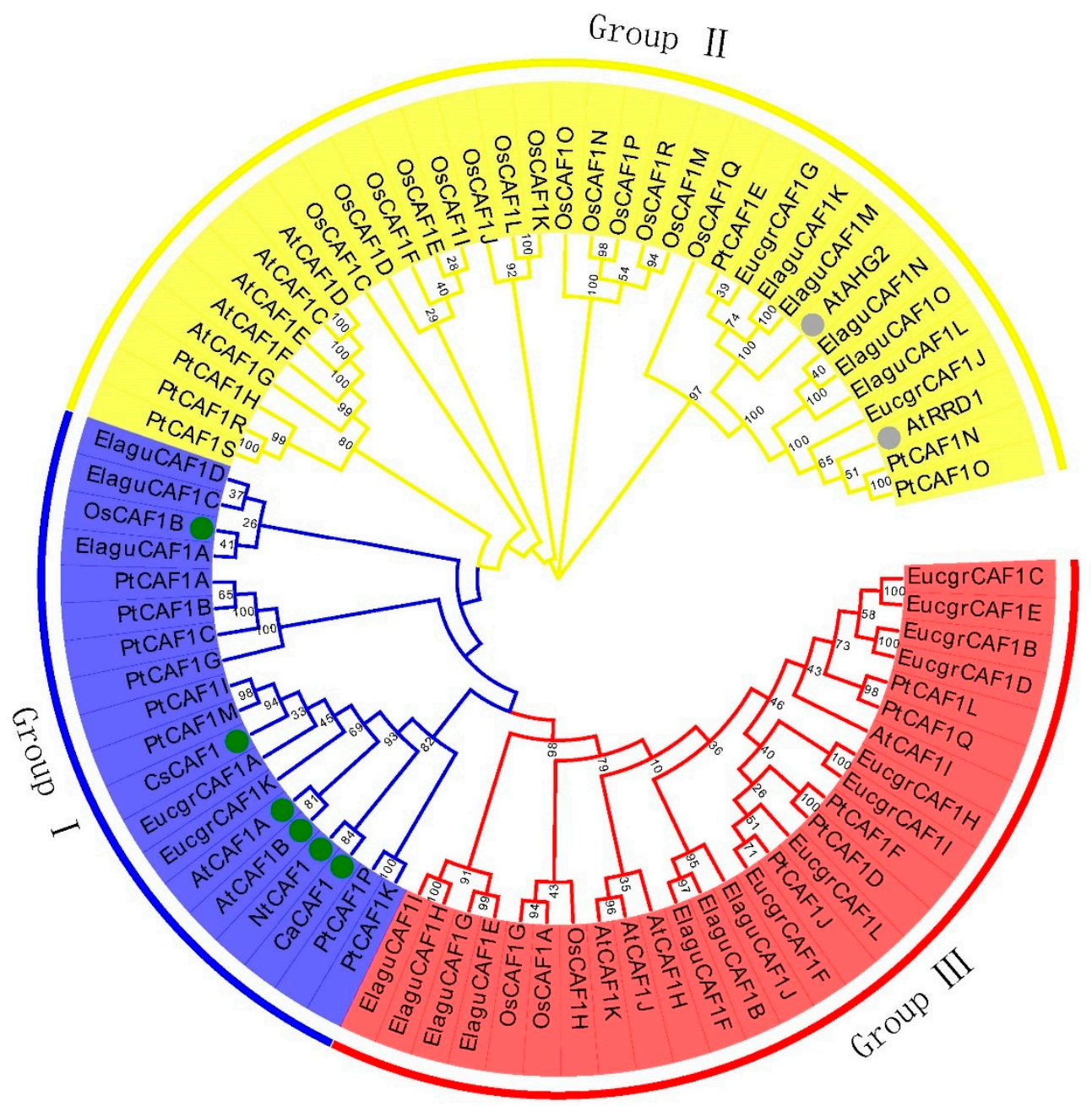

Figure 1. Phylogenetic relationship between P. trichocarpa and other plant species. Phylogenetic analyses of the PtCAF1 protein alignments of CAF1 domains among Oryza sativa, Arabidopsis thaliana, Eucalyptus grandis, Elaeis guineensis, Capsicum annuum, Citrus sinensis, and Nicotiana tabacum were performed with ClustalX2.1, and the phylogenetic tree was constructed using the NJ method with MEGA 7.0. The circle represents the gene whose function has been studied. The blue color denotes Group I, the yellow color denotes Group II, and the red color denotes Group III.

\subsection{Gene Structure and Motif Composition of PtCAF1 Gene Family}

The exon/intron structure pattern of the PtCAF1 genes and the conserved domain were studied according to their phylogenetic relationships. By comparing the genomic DNA sequences of the PtCAF1 genes, we obtained their intron and exon structures. The motifs of the 19 PtCAF1 genes were analyzed using online MEME software. According to the results of the MEME motif analysis, a schematic diagram was constructed to characterize the structure of the PtCAF1 proteins. In order to elucidate this result, 10 conserved motifs were found in the PtCAF1 proteins (Supplementary Materials, Figure S2). Moreover, we constructed a phylogenetic tree using the $P$. trichocarpa protein sequence and $A$. thaliana 
protein sequence, which is consistent with the aforementioned phylogenetic tree. The coding sequence of the PtCAF1 gene and the corresponding genomic DNA sequence were used to analyze the organization of exons/introns. The results are shown in Figure 2. There are differences in the number of introns between CAF1 genes. Group III and Group I exhibit a similar number of introns, ranging from 0 to 1 . However, in GroupII, PtCAF1 has 0 to 7 introns. The analysis of the gene structure and phylogenetic tree showed that the CAF1 gene between Group I and Group III experienced a series of evolutionary events leading to intron insertion, so they may have different functions. Group III genes contain eight motifs, and the types of motifs are the same. Group I genes contain similar motifs, with a maximum of eight motifs and a minimum of five motifs. Group II PtCAF1 has seven motifs and two motifs. These results indicate that the most closely related members have similar exon/intron structures and similar protein motifs. By studying the 19 PtCAF1 protein motifs, we found that the PtCAF1 protein sequence was highly conserved, and 10 motifs in the 19 PtCAF1 amino acid sequences were basically identical in the same family, especially in Group III. Studies of exon/intron structure have shown that most PtCAF1 from the same subfamily have similar exon numbers. The motif composition and sequence of all genes were consistent, which was highly conserved in evolution.

A

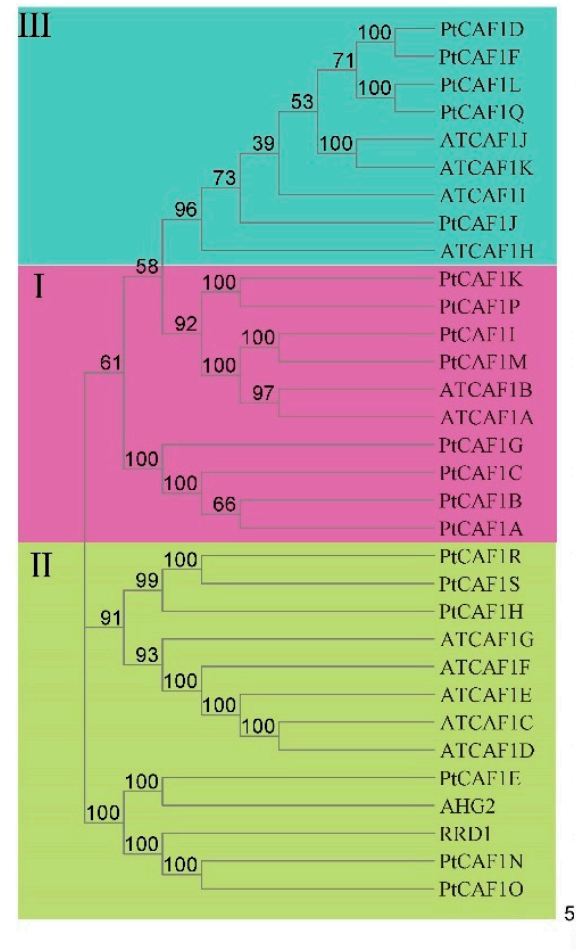

B

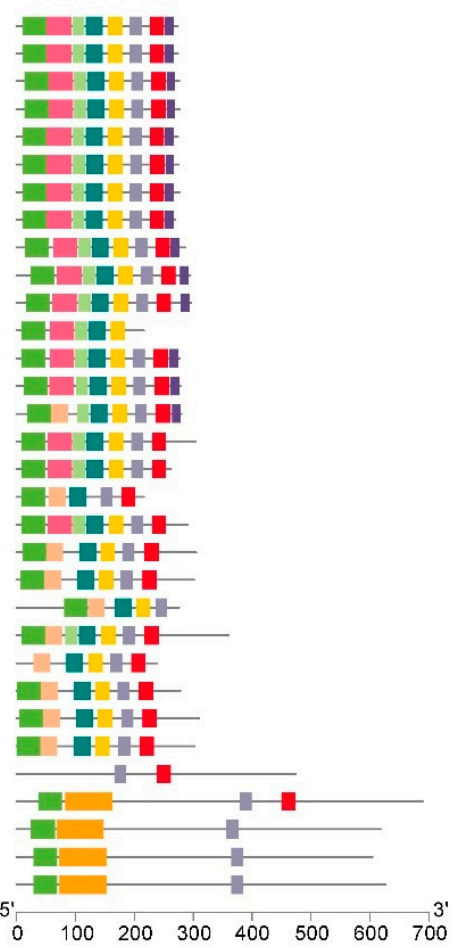

C

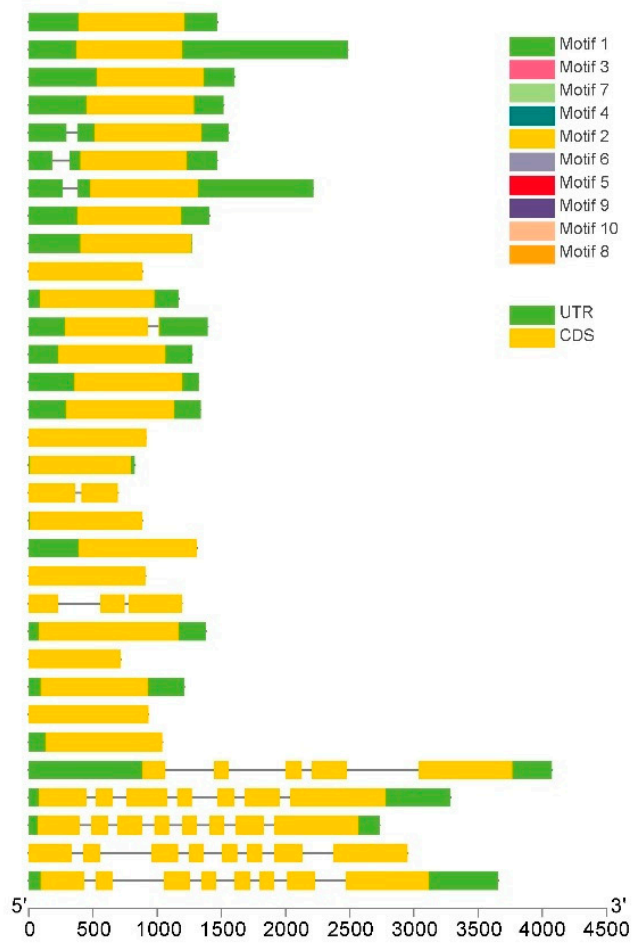

Figure 2. Phylogenetic relationships, gene structure, and architecture of conserved protein motifs in CAF1 from P. trichocarpa and A. thaliana. (UTR:Untranslated Regions. CDS: Coding DNA Sequence.) (A) The phylogenetic tree was constructed based on the full-length sequences of $P$. trichocarpa and A. thaliana CAF1 proteins using MEGA 7.0 software. (B) The motif composition of $P$. trichocarpa and A. thaliana CAF1 proteins. The motifs, numbers 1-10, are displayed in different colored boxes. The protein length can be estimated using the scale at the bottom. (C) Exon/intron structure of P. trichocarpa and A. thaliana CAF1 genes. Green boxes indicate untranslated $5^{\prime}$ and $3^{\prime}$ regions, yellow boxes indicate exons, and black lines indicate introns.

\subsection{Protein Structure Prediction of CAF1}

There is a close relationship between the spatial structure and function of the protein, because the function of the protein is realized by changing its spatial conformation. In order to further determine the spatial structure of CAF1, we used the SWISS-MODEL website 
(https:/ /swissmodel.expasy.org/ (accessed on 30 April 2021)) for homology modeling. All PtCAF1 and AtCAF1 proteins could be predicted as models, which indicates that they maintained their structural integrity during evolution, which plays an important role in their functions. In each branch, we selected one protein with the highest coverage from P. trichocarpa, O. sativa, and A. thaliana. Except for PtCAF1R (coverage 0.86) and OsCAF1B (coverage 0.8 ), the other confidence values are all higher than $90 \%$. The results are shown in the Figure 3. We also found that the spatial conformations of the P. trichocarpa, O. sativa, and $A$. thaliana proteins belonging to the same branch are highly similar.

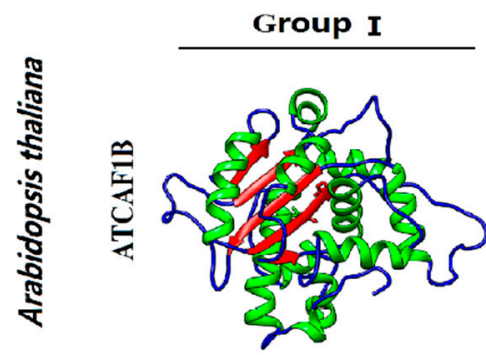

coverage 0.94

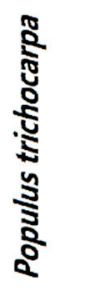
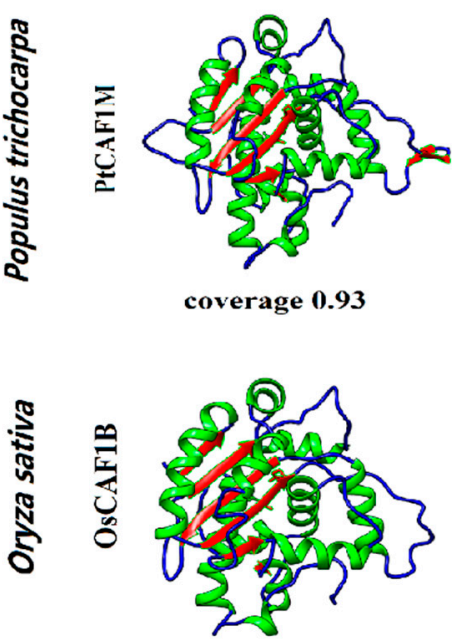

coverage 0.8

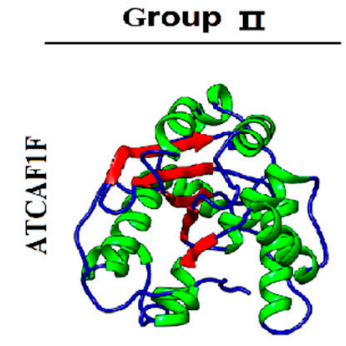

coverage 0.97

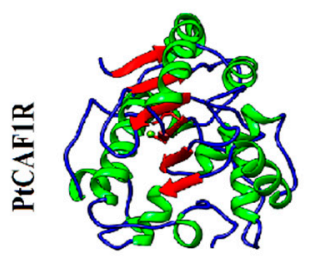

coverage 0.86

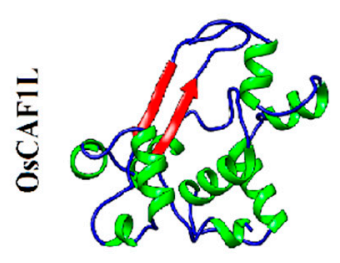

coverage 0.96

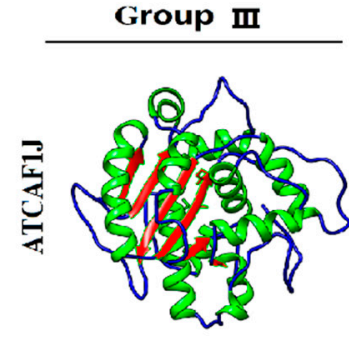

coverage 0.95

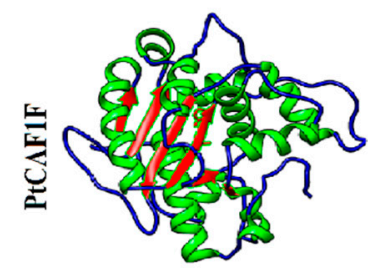

coverage 0.97

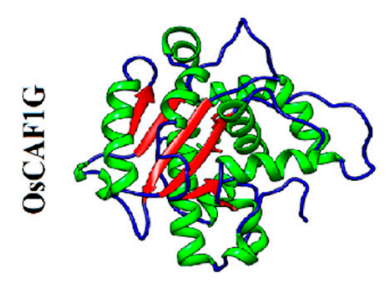

coverage 0.95

Figure 3. Prediction of the spatial structure of the CAF1 protein. The protein coil is blue, the helix is green, and the strand is red.

\subsection{Promoter cis-Element Analysis}

The $1.5 \mathrm{~kb}$ upstream sequences from PtCAF1 were programed in the PlantCARE server (Supplementary Materials, Table S1). As shown in Supplementary Materials, Figure S3, potential environmental factor-related cis-regulatory elements were predicted to be correlated with light and anaerobic induction response, which were most widely spread in the promoters of PtCAF1. The putative cis-acting regulatory DNA elements in PtCAF1 were identified. In addition to the basic elements (CAAT-box and TATA-box), most PtCAF1 genes had several cis-elements that were related to environmental stress signaling and phytohormone responses, such as LTR (low-temperature responsiveness), MBS (drought-inducibility), TATC-box (gibberellin-responsiveness), G-Box (light responsiveness), ABRE (the abscisic acid responsiveness), CGTCA-motif (the MeJA-responsiveness), ARE (anaerobic induction), MRE (light responsiveness), TC-rich repeats (defense and stress responsiveness), and TGACG-motif (the MeJA-responsiveness). It is well known that phytohormones, such as methyl jasmonate (MeJA) and abscisic acid (ABA), enable plants to adapt to abiotic stress. Thus, it is possible that most PtCAF1 genes respond to abiotic stress. 


\subsection{Chromosomal Distribution and Synteny Analysis of PtCAF1 Genes and Ka/Ks Calculation}

We identified 19 PtCAF1 gene sequences in the $P$. trichocarpa genome using bioinformatics methods, while there are 11 CAF1 genes in A. thaliana and 18 CAF1 genes in O. sativa. This may be due to the specific expansion of the pedigree and the loss of copy number during the evolution of the PtCAF1 gene family. Chromosome mapping of PtCAF1 genes was performed using the P. trichocarpa genome database. A total of 19 PtCAF1 genes were distributed on eight chromosomes. The largest number of PtCAF1 genes was found on Chromosome 1 (five genes), while Chromosomes 9 and 16 had the smallest number of PtCAF1 genes (one gene) (Figure 4). In addition, we analyzed the duplication events of PtCAF1 genes in the $P$. trichocarpa genome (Figure 5), because gene replication plays an important role in the occurrence of novel functions and gene expansion. Analyses of homologous protein families are of great significance in establishing the kinship of species and predicting the function of new protein sequences [34,35]. On the basis of the phylogenetic relationships, the duplication events were proposed to occur in the P. trichocarpa genome. Nine PtCAF1 genes (PtCAF1I/PtCAF1M, PtCAF1K/PtCAF1M, PtCAF1M/PtCAF1P, PtCAF1I/PtCAF1K, PtCAF1I/PtCAF1P, PtCAF1F/PtCAF1L, PtCAF1F/PtCAF1Q, PtCAF1D/PtCAF1F, PtCAF1J/ PtCAF1L, PtCAF1L/PtCAF1Q, PtCAF1D/PtCAF1K, PtCAF1J/PtCAF1Q, PtCAF1K/PtCAF1P, and PtCAF1D/PtCAF1Q) were clustered into 14 repeat event regions on Chromosomes 1 , $3,4,6,9,16$, and 18 of $P$. trichocarpa (Supplementary Materials, Table S2). There are three clusters on Chromosome 6, indicating a hot spot of PtCAF1 gene distribution. The results showed that part of the PtCAF1 genes might be generated by gene replication, and the fragment repeat event was the main driving force of PtCAF1 evolution. In order to further infer the phylogeny of PtCAF1 family in P. trichocarpa, we constructed two comparative collinearity maps of poplar based on the developmental mechanism, including one dicotyledon (A. thaliana) and one monocotyledon (O. sativa). Four PtCAF1 genes showed the same linear relationship with 2 AtCAF1 genes in A. thaliana, and 3 PtCAF1 genes showed the same linear relationship with one OsCAF1 gene in O. sativa. Interestingly, the PtCAF1L and PtCAF1Q genes showed the same linear relationship in $A$. thaliana. It is suggested that these genes play an important role in the evolution of the PtCAF1 gene family in P. trichocarpa.

In order to understand the evolutionary constraints of the PtCAF1 family, we calculated the nonsynonymous to synonymous substitution ratios $(\mathrm{Ka} / \mathrm{Ks})$ of $P t C A F 1$ gene pairs (Table 2). All fragments and repeated PtCAF1 gene pairs and most of the homologous PtCAF1 genes had a Ka/Ks of < 1 except for PtCAF1C \& PtCAF1G, which indicated that the PtCAF1 gene family of poplar may have experienced a strong purification selection pressure during its evolution. The divergence time of the paralogous gene pairs of PtCAF1F \& PtCAF1L and PtCAF1C \& PtCAF1G were estimated to be about 200 million years ago, respectively. In brief, some PtCAF1 genes might have been produced by gene replication, and these replication events were the main driving force of PtCAF1 evolution.

Table 2. $\mathrm{Ka} / \mathrm{Ks}$ analysis of the PtCAF1 gene pairs duplication.

\begin{tabular}{ccccccc}
\hline Sequence & Ka & Ks & Ka/Ks & $p$-Value (Fisher) & Length & Time (Mya) \\
\hline PtCAF1D \& PtCAF1F & 0.016708 & 0.306409 & 0.05453 & $1.36 \times 10^{-22}$ & 822 & 102.1363 \\
PtCAF1A \& PtCAF1C & 0.014891 & 0.019054 & 0.781482 & 0.429132 & 780 & 6.351433 \\
PtCAF1C \& PtCAF1G & 1.01436 & 0.952687 & 1.06473 & 0.247271 & 723 & 317.5623 \\
PtCAF1A \& PtCAF1G & 0.12254 & 0.316388 & 0.387308 & $5.67 \times 10^{-8}$ & 873 & 105.4627 \\
PtCAF1F \& PtCAF1L & 0.082084 & 2.14757 & 0.038222 & $4.1 \times 10^{-125}$ & 819 & 715.8567 \\
PtCAF1K \& PtCAF1P & 0.049893 & 0.434185 & 0.114912 & $8.21 \times 10^{-28}$ & 858 & 144.7283 \\
PtCAF1N \& PtCAF1O & 0.008309 & 0.01364 & 0.609174 & 0.239107 & 1797 & 4.546667 \\
PtCAF1L \& PtCAF1Q & 0.034304 & 0.337114 & 0.101757 & $2.86 \times 10^{-20}$ & 831 & 112.3713 \\
PtCAF1R \& PtCAF1S & 0.028137 & 0.084529 & 0.332862 & 0.000561 & 906 & 28.17643 \\
\hline
\end{tabular}

Note: Ka: The ratio of the number of nonsynonymous substitutions per nonsynonymous site. Ks: the number of synonymous substitutions per synonymous site. Ka/Ks: the nonsynonymous to synonymous substitution ratios. Mya: million years ago. 


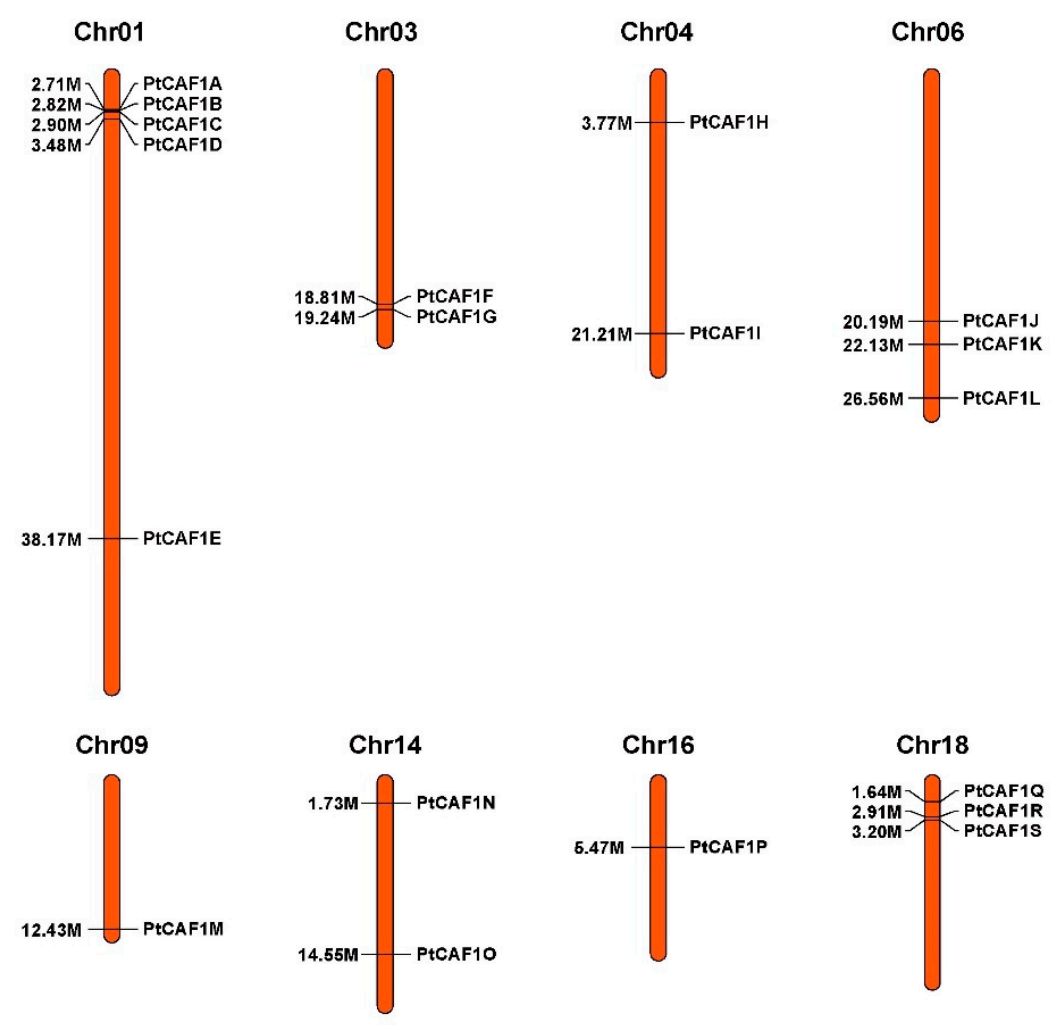

Figure 4. Chromosomal distribution of the 19 PtCAF1 genes. The scale of the chromosome is in megabases $(\mathrm{Mb})$. The chromosome number is indicated at the top of each chromosome.

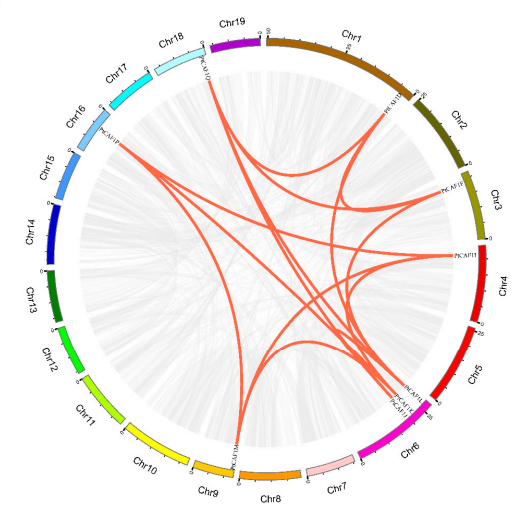

B

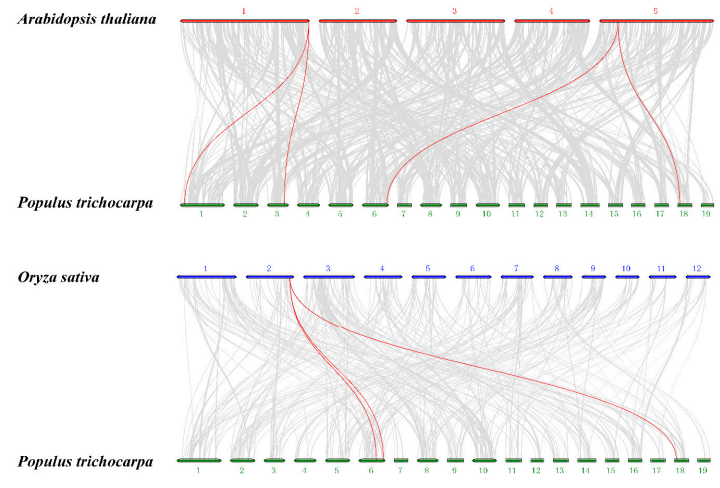

Figure 5. Duplication event analysis of CAF1 genes and comparative synteny analysis among P. trichocarpa, A. thaliana, and O. sativa. Gray lines in the background indicate the collinear blocks within P. trichocarpa and other plant genomes, while red lines highlight syntenic PtCAF1 gene pairs. (A) The data were derived from the Plant Genome Duplication Database, and 14 couples of duplicated PtCAF1 genes were anchored to corresponding positions on $P$. trichocarpa chromosomes using the CIRCOS program. (B) Synteny between P. trichocarpa and A. thaliana or between P. trichocarpa and $O$. sativa was anchored to the corresponding position on specific chromosomes using the CIRCOS program. P. trichocarpa chromosomes are depicted as green segments, and A. thaliana and O. sativa are shown in red and blue, respectively. The size of chromosomes was consistent with the actual pseudo-chromosome size.

\subsection{Expression Patterns of PtCAF1 Genes in Different Plant Tissues}

In order to investigate the function of the PtCAF1 gene family in different tissues, the gene expression data of the 19 PtCAF1 genes were obtained from the GEO datasets (NCBI 
GEO accession GSE81077). As shown in Figure 6, RNA-Seq and qRT-PCR data generated in the heatmap demonstrated a significant expression variation of PtCAF1 in all vegetative and reproductive tissues (also see the analysis data in Supplementary Materials, Data S1). The transcriptional data showed that the expression of the PtCAF1 gene in different tissues was different, and the expression of the PtCAF1E, PtCAF1G, PtCAF1I, PtCAF1K, PtCAF1L, PtCAF1M, PtCAF1O, PtCAF1P, PtCAF1Q, and PtCAF1S genes in the root was highest. Except for $P t C A F 1 K$, the expression of the aforementioned geens in the leaves was the lowest. The expression of the PtCAF1A, PtCAF1B, and PtCAF1J genes in the root was very low. The expression of PtCAF1J and PtCAF1H in the leaves was highest, and the expression of PtCAF1A, PtCAF1B, PtCAF1C, PtCAF1F, PtCAF1N, and PtCAF1R in the stem was also highest.

A

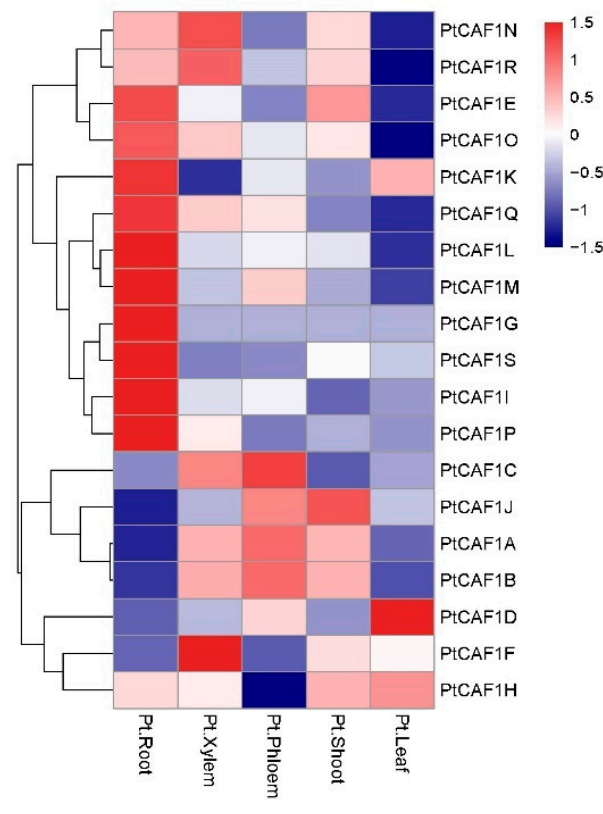

B

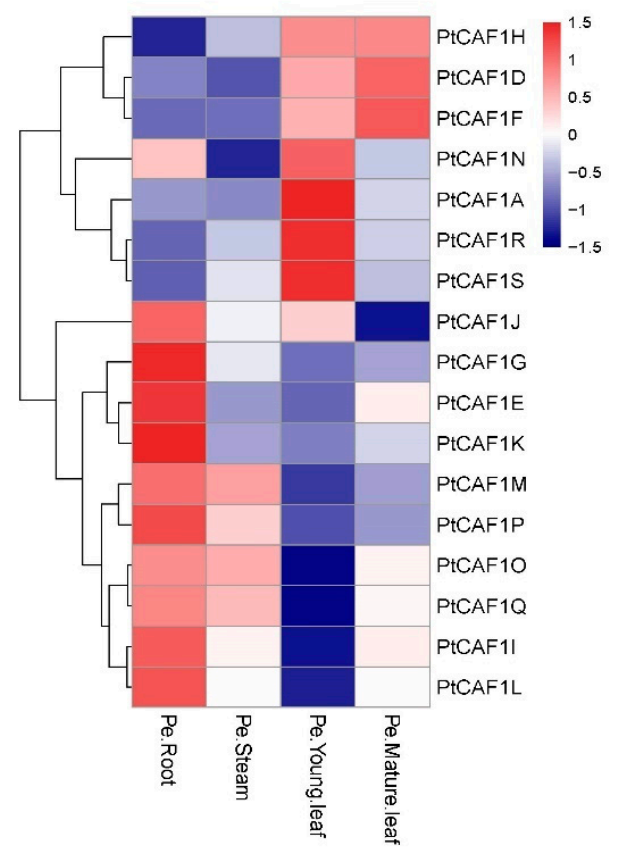

Figure 6. Tissue-specific gene expression of PtCAF1 genes. The expression patterns of PtCAF1 genes in leaf, root, and stem tissues were examined by RNA-Seq and qRT-PCR data. The heatmap was created based on the log2-transformed values of the relative expression levels of the PtCAF1 genes. (A) PtCAF1 gene family expression in different $P$. trichocarpa tissues and organs. (B) Tissue-specific expression of PtCAF1 in 'Nanlin $895^{\prime}$. Expression levels are represented in various colors, with red indicating higher expression levels and navy indicating lower expression levels.

To further study the CAF1 expression in different tissues of poplar, we studied the expression of the 19 CAF1 genes in 'Nanlin895' poplar under normal growth conditions, and the transcription levels of the 19 CAF1 genes in the roots, stems, young leaves, and mature leaves of 'Nanlin895' poplar were detected by quantitative reverse transcription PCR (qRT-PCR). Most PtCAF1 genes were expressed in a wide range of tissues, and only two genes (PtCAF1B and PtCAF1C) could not be detected in the experiment. The qRT-PCR results showed that $17 P t C A F 1$ genes were expressed in four tissues. The results showed that the expression levels of PtCAF1E, PtCAF1G, PtCAF1I, PtCAF1J, PtCAF1K, PtCAF1L, PtCAF1M, PtCAF1O, PtCAF1P, and PtCAF1Q were higher in the roots of 'Nanlin895' poplar, which was similar to the transcriptome data. The expression of PtCAF1A, PtCAF1N, and $P t C A F 1 R$ in young leaves was higher than in mature leaves. The results showed that the expression of PtCAF1 genes varied in different tissues, suggesting that the PtCAF1 genes had multiple functions in poplar growth. 


\subsection{Expression Profiling of PtCAF1 in Response to Different Treatments}

Poplar plays a significant role in the chemical industry in China. However, the growth of poplar is limited by environmental pressures such as salt, drought, and pathogenic bacteria [36,37]. In order to confirm whether the expression of the PtCAF1 gene was affected by different stresses, 17 PtCAF1 members were selected from 19 PtCAF1 genes. We carried out various stress and hormone treatments on 'Nanlin895'. The expression of the PtCAF1 gene was detected by qRT-PCR under different conditions (see Figure 7 and the analysis data in Supplementary Materials, Date S1). The results showed that the expressions of 17 genes were up-regulated under $\mathrm{ABA}$ and salt treatment, among which PtCAF1E, PtCAF1I, PtCAF1L, PtCAF1M, PtCAF1N, and PtCAF1Q were significantly increased after ABA treatment for $12 \mathrm{~h}$, and the PtCAF1 family after $6 \mathrm{~h}$ of salt treatment. In $\mathrm{H} 2 \mathrm{O} 2$ treatment, most $P t C A F 1$ gene expression was up-regulated, and the expression of the PtCAF1A, PtCAF1G, PtCAF1P, PtCAF1O, and PtCAF1R genes was significantly increased after $12 \mathrm{~h}$ of treatment. In the PEG treatment, the expression of the PtCAF1I, PtCAF1K, PtCAF1L, PtCAF1M, and PtCAF1Q genes was increased considerably. The expression of the PtCAF1A, PtCAF1O, PtCAF1P, and PtCAF1S genes was significantly increased as a result of cold treatment. In the wound treatment, only the expression of the PtCAF1F gene was significantly increased. Interestingly, most genes reached the highest expression in 3 days of Marssonina brunnea treatment, and they were down-regulated at 5 days of $M$. brunnea treatment. At the same time, the changes in gene expression were not apparent in the process of $3 \mathrm{~h}$ to $6 \mathrm{~h}$ of JA and SA treatment; only PtCAF1D, PtCAF1F, PtCAF1L, and PtCAF1Q fluctuated. The results showed that some PtCAF1 genes were induced or inhibited by multiple treatments. For example, the PtCAF1D, PtCAF1F, PtCAF1J, PtCAF1L, and PtCAF1Q genes in Group III were significantly up-regulated in all treatments and remained at a high level. In contrast, one treatment induced multiple PtCAF1 genes at the same time. For example, 17 genes were significantly up-regulated in response to salt treatment.

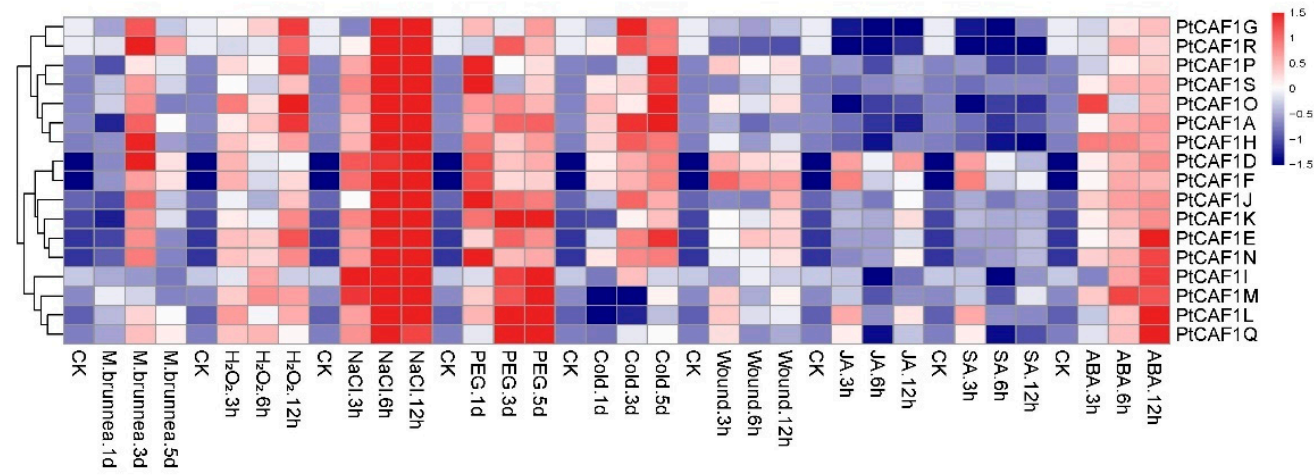

Figure 7. The expression profiles of the PtCAF1 gene family in poplar under abiotic or biotic stresses and phytohormone treatments. The heatmaps were constructed using the $\mathrm{R}$ package based on the expression datasets. Expression levels are represented in various colors, with red indicating higher expression levels and navy indicating lower expression levels.

\section{Discussion}

The CAF1 gene is a relatively conservative gene that exists in a wide range of animals and plants. It has been extensively studied in yeast [38]. Thus far, the CAF1 gene has been amply studied in animals, but there is a lack research in plants [39]. In this study, we analyzed the PtCAF1 gene family in P. trichocarpa using phytozome v12.1. We identified 19 PtCAF1 genes in P. trichocarpa. Moreover, we analyzed the structure and the chromosome location, performed a phylogenetic analysis, and checked the stress response mode of the PtCAF1 gene family. These results lay a foundation for the further study of the PtCAF1 gene. In the present study, 19 PtCAF1 genes were identified, mainly distributed on eight chromosomes of $P$. trichocarpa. According to their chromosome location, they were named 
PtCAF1A to PtCAF1S. In order to explore the evolution of the CAF1 gene family, the evolutionary tree of the $C A F 1$ gene was analyzed. We divided the poplar gene family into three group by distributing the PtCAF1 gene family with $A$. thaliana and O. sativa. Most of the genes belong to Group II. Interestingly, by searching for Pham in the $A$. thaliana genome, it was found that, in addition to the 11 AtCAF1 genes that were identified, two genes (AtAHG2 and AtRRD1) have the CAF1 domain. Furthermore, the results of the Pham search in the $O$. sativa genome are the same as those of previous studies. It was found that AtAHG2 encodes a poly(A)-specific ribonuclease (AtPARN), which participates in the degradation of mRNA in plants [40]. In addition, SA and ABA play a key role in the external stress response [41]. Various results showed that the mutant ahg2-1 had higher endogenous SA, ABA, and various stress reactions in A. thaliana [42]. Moreover, various studies indicated that $A t R R D 1$ could participate in cell division during early lateral root organogenesis [43]. PtCAF1E, PtCAF1N, PtCAF1O, AtAHG2, and AtRRD1 were in the same class, and PtCAF1E, PtCAF1N, and PtCAF1O were up-regulated to different degrees in the late response to stress. Therefore, PtCAF1E, PtCAF1N, and PtCAF1O may play an essential role in stress resistance in poplar. In addition, the subcellular localization of the gene is directly related to its function. The main function of the CAF1 gene is to participate in the degradation of mRNA poly A, and 13 genes were found in the cytoplasm in the subcellular localization results. Other genes were located in the nucleus and chloroplast.

The study of exons and introns is helpful to understand the structural and functional differences of genes. Their acquisition or loss may be caused by gene duplication between different chromosomes [44,45]. In this study, we compared the protein sequences of CAF1 members in $P$. trichocarpa and $A$. thaliana, and analyzed their phylogeny and gene structure. It was found that the CAF1 family belonging to the same group usually has a similar motif composition and exon/intron structure. This indicates that they may have similar functions. Furthermore, specific sequence motifs in each group can endow the CAF1 protein with specific functions, and the differences in these characteristics in different groups indicate the diversity of PtCAF1 gene functions. Interestingly, the number of motifs of certain genes in Group II was significantly lower than that of other genes. These genes may have changed dramatically in evolution. We also found that the 19 PtCAF1 genes contained different exons and introns, which indicates that there was a certain diversity among these three group genes. Regarding the distribution of introns and exons of the PtCAF1 gene and AtCAF1 gene, most Group I and Group III genes have no introns, and they contain at most one intron. The number of introns and exons of the PtCAF1 genes in Group II is quite different: they contain seven introns or eight exons at most. The great difference in gene structure between different species indicates that significant changes took place in the long-term evolution of the poplar genome; however, the similarity of the gene structure in the same branch is very high, which indicates that the genes in the same group are very conservative as regards evolution.

Cis-elements play a key role in plant stress responses. For example, ABA-responsive elements (ABRE) respond to ABA hormone treatment, drought, and salt stress. LTR is involved in the plant response to low temperature. The TCA element and CGTCA motif were correlated with the expression level of MeJA and SA, respectively [46,47]. PtCAF1 contains a variety of defense and stress response-related elements, indicating that PtCAF1 plays an important role in biotic and abiotic stress. Plants have complex signal transduction networks in response to various external reactions, and they synthesize small hormones, such as MeJA and ABA, as signal molecules for the plant defense response [48]. A great deal of cis-elements responding to environmental stress were found in the promoter regions of the 19 PtCAF1 genes, such as ABRE (the abiotic acid response), MBS (deficit induction), ARE (anaerobic induction), and TC-rich repeats (defense and stress response). ABRE, as an ABA-response element, is involved in regulating the drought response of poplar [49]. PtCAF1D, PtCAF1L, and PtCAF1P contained more than two ABRE elements, which may indicate that PtCAF1D, PtCAF1L, and PtCAF1P were involved in the response to drought stress. Many LTR (low-temperature responses) were found in the promoter region of 
PtCAF1O, which indicated that LTR is involved in the low temperature response of poplar. The results showed that the PtCAF1 gene might participate in the response of poplar to environmental stress through ABA and other signaling channels.

Indeed, previous studies have reported that tandem and fragment replication events are important factors in the expansion and evolution of gene families. The occurrence of repetitive events in the genome helps plants adapt to the changing environment and evolve more smoothly [50-53]. Gene doubling events mainly include tandem replication, fragment replication, and reverse transcription transposition, which are important ways of gene family expansion [54]. Most of the homologous PtCAF1 gene pairs had a Ka/Ks of $<1$, indicating that the PtCAF1 gene may have been under selection pressure during evolution. Gene replication also plays an important role in promoting the evolution of the genome. Repetitive genes provide templates for new genes, which have new functions. There were 14 repeat event regions on seven chromosomes of $P$. trichocarpa, which indicates that repeat events played a key role in the expansion of the PtCAF1 gene. The fragment replication of PtCAF1 in P. trichocarpa mainly occurred in Group I and Group III. The collinearity analysis of $P$. trichocarpa with $A$. thaliana and $O$. sativa showed that gene replication mainly occurred in Group III, and the genetic relationship between $P$. trichocarpa and A. thaliana might be closer. The degree of differential expression of duplicate gene pairs is positively correlated with the time of differentiation: the longer the duplication, the more obvious the differentiation of the expression [55-57]. In this study, the PtCAF1F \& PtCAF1L divergence time is the longest, and there are also various differences in the degree of response to different environments in later stages.

We analyzed the PtCAF1 gene expression in four different tissues of 'Nanlin895'. The results showed that PtCAF1 gene expression was different in the four tissues. Most of the PtCAF1 genes were highly expressed in the roots, while a small number of PtCAF1 genes were expressed in the stems. This is consistent with the transcriptional data of $P$. trichocarpa. However, there are also differences. This may be due to the difference of some genes between P. trichocarpa and 'Nanlin895'. The high expression level of the PtCAF1 gene in the roots may reflect that the PtCAF1 family plays an important role in responses to abiotic and biotic stresses from underground. Interestingly, the expression levels of 17 genes were high in the roots and leaves, but low in the stems. It may be that PtCAF1 participates in the response of poplar to environmental stress, which leads to its unique expression pattern.

The PtCAF1 gene plays an important role in regulating plant development, and giving plants tolerance to abiotic stresses (including salt, drought, heat, cold, and wound) [58]. In view of the importance of poplar in abiotic stress and the key role of the PtCAF1 gene in physiological processes and stress responses, gene-specific expression usually reflects its corresponding function $[59,60]$. In this paper, the expression of PtCAF1 in different environments was analyzed by qRT-PCR. Seventeen PtCAF1 genes were differentially expressed in different environments, indicating that the PtCAF1 gene may be involved in the response to stresses. It has been reported that PtCAF1 is responsive to biotic stress in O. sativa, A. thaliana, and C. sinensis. Moreover, we found that most PtCAF1 genes were significantly up-regulated after M. brunnea treatment for 3 days, with only PtCAF1I and PtCAF1M demonstrating no significant change. The results showed that the PtCAF1 gene played an important role in responses to biological stress in poplar. Various recent studies report that the plant CAF1 gene is mainly involved in responses to biotic stress. There is a lack of research on its response to abiotic stress, though various studies report that $C A F 1$ plays an important role in responses to low temperature stress in $O$. sativa and responses to wound stress in A. thaliana and O. sativa $[27,29,61]$. In this study, 17 genes were upregulated after salt stress, indicating that PtCAF1 plays a key role in responses to salt stress. In PEG, $\mathrm{H}_{2} \mathrm{O}_{2}$, and low temperature treatment, most genes were up-regulated. In addition, $\mathrm{ABA}$ plays a leading role in the regulation of plant adaptation to abiotic stresses, because ABA triggers many gene responses in plants and helps plants respond to environmental stimuli such as cold, drought, and salt [62-65]. In our study, after $12 \mathrm{~h}$ of ABA treatment, 17 PtCAF1 genes were up-regulated to varying degrees. It may be that the PtCAF1 gene 
is involved in the ABA metabolic regulation pathway, which causes PtCAF1 to respond to abiotic stresses. In this study, the expression of the PtCAF1 gene under cold, PEG, $M$. brunnea, $\mathrm{H}_{2} \mathrm{O}_{2}$, and salt stress differed. In addition, different stress can activate the same genes in different signaling pathways, which may be due to the production of common signaling components such as ABA or calcium caused by different stress stimuli $[66,67]$.

As a model woody plant, poplar has a different life history to $A$. thaliana and O. sativa and may be more complex in terms of development and its gene regulation network [68-70]. Drought stress, salt stress, oxidation, plant pathogenic bacteria, and other factors seriously damage the growth and development of poplar. There is a lot of evidence to suggest that PtCAF1 regulates plant growth and certain physiological processes and plays an important role in the response of the biotic and abiotic stress-induced defense signaling pathways $[5,23]$. Certain recent studies demonstrate that miRNA, which is a single stranded small RNA encoded by the higher eukaryotic genome, is also involved in mRNA degradation and in regulating the expression of more than one third of genes. It is an important component of the gene expression regulatory network and has an impact on expression levels [71]. In mammals, miRNA and CAF1 can accelerate the deadenylation of the poly(A) tail of the target mRNA [72]. Furthermore, miR2275 and CAF1 were found to be involved in early meiosis in male sterile wheat $[5,73]$. Therefore, miRNA and CAF1 may regulate the expression of stress resistance genes in poplar. PtCAF1 can participate in multiple abiotic stresses, and interaction with other proteins also plays a crucial role. In terms of potential applications, the PtCAF1 gene has potential value in stress resistance, and targeting these genes may improve abiotic and biotic stress responses. In conclusion, the study provides an analysis of the PtCAF1 gene family in P. trichocarpa, which provides a basis for the study of the potential function of the PtCAF1 gene in poplar. These analyses can help to screen candidate PtCAF1 genes for functional identification and provide a theoretical basis for the genetic improvement of the agronomic characteristics and environmental resistance of poplar.

\section{Materials and Methods}

\subsection{Identification and Characterization of the CAF1 Family in P. trichocarpa}

The complete $P$. trichocarpa protein sequences were downloaded from the JGI (Phytozome v12.1, https:/ / phytozome.jgi.doe.gov/pz/portal.html (accessed on 30 April 2021)). To identify P. trichocarpa CAF1 candidates, the Hidden Markov model (HMM) file corresponding to the CAF1 domain (PF04857) was downloaded from the Pfam database (http:/ / pfam.xfam.org/ (accessed on 30 April 2021)). HMMER 3.0 was used to search the CAF1 gene from the P. trichocarpa genome database. The default parameter was determined and the cutoff was $1.2 \times 10^{-28}$. We also used it as the query to search the $P$. trichocarpa protein sequence data. To avoid missing probable CAF1 members, the $P$. trichocarpa genome was used as the query to perform a BLAST search in the A. thaliana and O. sativa CAF1 gene sequences, with a cutoff E-value of $\leq 10^{-10}$. The output putative $C A F 1$ protein sequences were submitted to the Pfam protein family database, SMART (Simple Modular Architecture Research Tool, http:/ / smart.embl-heidelberg.de/ (accessed on 30 April 2021)), and CDD (Conserved Domain Database, https:/ /www.ncbi.nlm.nih.gov/Structure/bwrpsb/bwrpsb.cgi (accessed on 30 April 2021)) to confirm the conserved CAF1 domain.

\subsection{Sequence Analysis}

All the PtCAF1 gene sequences were submitted to ExPASy (http:/ / web.expasy.org/ protparam/(accessed on 30 April 2021)) to find the number of amino acids, molecular weights (MW), and theoretical isoelectric points (PI). The PtCAF1 subcellular targeting sites were identified using WOLF PSORT (https:/ / wolfpsort.hgc.jp / (accessed on 30 April 2021)). The chromosomal locations and intron numbers of PtCAF1 were acquired through the P. trichocarpa genomic database. The motifs of PtCAF1 proteins sequences were identified by MEME (http:/meme.nbcr.net/meme/intro.html (accessed on 30 April 2021)). The position information of the CAF1 domains was obtained from the Pfam database, and the PtCAF1 
structure information was parsed from the General Feature Format files of $P$. trichocarpa using Perl script. Then, the exon and intron of the PtCAF1 genes were identified on GSDS (the Gene Structure Display Server, http:/ / gsds.cbi.pku.edu.cn/ (accessed on 30 April 2021)). In order to identify the cis-acting regulatory elements in promoters of the PtCAF1 genes, 1500 bp upstream regions of the CDS were used to search the Plant Cisacting Regulatory Elements (PlantCARE) database (http:/ /bioinformatics.psb.ugent.be/ webtools/plantcare/html/ (accessed on 30 April 2021)).

\subsection{Multiple Sequence Alignment and Phylogenetic Analysis of CAF1 Proteins}

Multiple sequence alignment was conducted using the Clustal X2.1 software with default settings. The CAF1 protein sequences of A. thaliana, O. sativa, C. annuum, C. sinensis, N. tabacum, yeast (SpCAF1), human (HsCNOT7), mouse, P. trichocarpa, and Z. mays (ZmCAF1) were used for multiple sequence alignment. A phylogenetic tree was constructed in the Molecular Evolutionary Genetics Analysis (MEGA7) software (https:// www.megasoftware.net/ (accessed on 30 April 2021)). According to the CAF1 conserved domain, PtCAF1 genes were divided into different groups. The $C A F 1$ protein sequences for O. sativa, A. thaliana, E. grandis, E. guineensis, C. annuum, C. sinensis, $P$. trichocarpa, and $N$. tabacum were used for the phylogenetic tree analysis. The CAF1 protein sequences of A. thaliana, E. grandis [74], and O. sativa were downloaded from the JGI gene catalog (Phytozome v12.1, https:/ / phytozome.jgi.doe.gov/pz/portal.html (accessed on 30 April 2021)). The $C$. annuum (CaCAF1), C. sinensis, yeast (SpCAF1), mouse, human, E. guineensis, and Z. mays CAF1 protein sequences were downloaded from the National Center for Biotechnology Information (https:/ /www.ncbi.nlm.nih.gov/ (accessed on 30 April 2021)). All sequences of different species' CAF1 genes are listed in Supplementary Materials, Table S3. Moreover, all species' CAF1 protein sequences are listed in Supplementary Materials, File S1. The phylogenetic trees were constructed using the neighbor-joining $(\mathrm{NJ})$ method, the gaps/missing data treatment method was used for partial deletion, the specific model/method was the Poisson model, the number of bootstrap replications was 1000, and the site coverage cutoff was 0.95 [75].

\subsection{Prediction of Protein Spatial Structure}

In order to predict the spatial structure of proteins, the SWISS-MODEL website (https: / swissmodel.expasy.org/ (accessed on 30 April 2021)) was used to analyze the CAF1 protein structures of $P$. trichocarpa, $O$. sativa, and A. thaliana. The protein spatial structure was constructed using the UCSF Chimera software (https:/ / www.cgl.ucsf.edu/ chimera/ (accessed on 30 April 2021)).

\subsection{Chromosomal Locations, Gene Duplication of PtCAF1 Genes, and Calculation of Ka/Ks}

The chromosomal positions of the PtCAF1 genes were obtained from the P. trichocarpa genome. MapChart was used for the mapping of PtCAF1 chromosomal positions and distances. In order to understand the evolutionary constraints of PtCAF1, we calculated the $\mathrm{Ka} / \mathrm{Ks}$ ratio of the PtCAF1 gene pairs. Hits with an E-value of $<10^{-5}$ and a homology greater than $80 \%$ were considered significant. For estimating the Ks and Ka substitution rates, the amino acids of paralogous and orthologous PtCAF1 proteins were analyzed using the $\mathrm{Ka} / \mathrm{Ks}$ calculator. The divergence time was estimated with the formula $\mathrm{T}=\mathrm{Ks} / 2 \mathrm{r}$. The $\mathrm{r}$ was taken to be $1.5 \times 10^{-8}$ synonymous substitutions per site per year for dicotyledonous plants [76]. For the homology analysis, we studied the repetitive events of PtCAF1 genes in the A. thaliana and O. sativa genomes from the plant genome duplication database. The analysis of gene replication events was conducted with multiple collinear scanning toolkits (MCScanX). Then, the synteny blocks were illustrated with the CIRCOS software (http: // circos.ca/software/download/circos (accessed on 30 April 2021)) between P. trichocarpa genes in A. thaliana and O. sativa. 


\subsection{Transcriptional Analyses}

The P. trichocarpa $\mathrm{cv}$. and P. deltoides $\times$ P. euramericana $\mathrm{cv}$. 'Nanlin895' were used in this study. P. trichocarpa and 'Nanlin $895^{\prime}$ were cultured in an artificial climate chamber with a $16 \mathrm{~h} / 8 \mathrm{~h}$ photoperiod in 1/2 Murashige and Skoog (MS) medium ( $\mathrm{pH}$ 5.8) at a temperature of $23 \pm 1{ }^{\circ} \mathrm{C}$. RNA was extracted from young and mature leaves, stems, and roots using a Plant RNA Extraction Kit (Biomiga, Inc., San Diego, CA, USA). M. brunnea was cultured in potato medium (PDA) at $25^{\circ} \mathrm{C}$. WT poplars grown for 3 months were treated with $200 \mathrm{mM}$ $\mathrm{ABA}, 200 \mathrm{mM} \mathrm{SA}, 200 \mathrm{~m} \mathrm{JA}, 2 \mathrm{mM} \mathrm{H}_{2} \mathrm{O}_{2}$, and $200 \mathrm{mM} \mathrm{NaCl}$, and samples were taken at 0 , 3,6 , and $12 \mathrm{~h}$. They were also treated with Macrogol 6000 (PEG6000), M. brunnea, at $4{ }^{\circ} \mathrm{C}$ (cold stress), and sampled at $0,1,3$, and 5 days. We also wounded leaves at $23{ }^{\circ} \mathrm{C}$, and samples were taken at $0,3,6$, and $12 \mathrm{~h}$. RNA was then extracted from the leaves using a Plant RNA Extraction Kit (Biomiga, Inc.).

Premier 5 was used to design primers specific to the PtCAF1 genes (Supplementary Materials, Table S4). Total RNA was extracted using a Plant RNA Extraction Kit (Biomiga, Inc.). The cDNA was obtained using a First Strand cDNA Synthesis Kit (TaKaRa, Shiga, Japan). First-strand cDNA in the reactions comprised a $500 \mathrm{ng}$ RNA template, and all the procedures were carried out according to the instructions provided by the kit. Before the qRT-PCR analysis, the template was diluted 10 times. The relative expression level of the gene was calculated by comparing the average of EF1 $\alpha$ (Potri.006G130900.1) and PtActin (Potri.019G006700.1) internal reference genes. The qRT-PCR reaction system included $10 \mu \mathrm{L}$ SYBR Green, $1 \mu \mathrm{L}$ forward primer, $1 \mu \mathrm{L}$ reverse primer, $1 \mu \mathrm{L}$ cDNA, and $7 \mu \mathrm{L} \mathrm{ddH}_{2} 0$. qRT-PCR involved 40 cycles of pre-denaturation at $98^{\circ} \mathrm{C}$ for $10 \mathrm{~min}$, denaturation at $95^{\circ} \mathrm{C}$ for $10 \mathrm{~s}$, and annealing at $60^{\circ} \mathrm{C}$ for $30 \mathrm{~s}$. The method of $2^{-\Delta \Delta C T}$ was used to analyze the data in this paper [77].

\section{Conclusions}

In this study, we identified 19 poplar PtCAF1 genes. The classification, gene structure, and evolutionary characteristics of the PtCAF1 genes in poplar showed that CAF1 was relatively conservative in terms of the evolutionary process. The differential expression of the PtCAF1 genes in poplar tissues showed that they played different roles in the development of poplar, and many genes showed tissue-specific expression patterns. In addition, a PtCAF1 gene expression analysis showed that certain genes were significantly up-regulated or down-regulated under biotic and abiotic stresses. Our results also revealed the differences in the expression of PtCAF1 induced by biotic and abiotic stresses in poplar, indicating that these PtCAF1 genes are involved in abiotic stress tolerance. In conclusion, our study established the structural and functional framework of the PtCAF1 protein. Although poplar genome sequencing has been carried out for many years, the identification and function of poplar stress-related genes still remains to be studied. Our results will be helpful for the further study of the important role of the PtCAF1 gene in poplar stress responses. It may also be utilized in molecular breeding programs for poplar stress tolerance.

Supplementary Materials: The following are available online at https:/ /www.mdpi.com/article/10 .3390/plants10050981/s1, File S1: Amino acid sequences of PtCAF1 proteins in Arabidopsis thaliana, Oryza sativa, Populus trichocarpa, Zea mays Eucalyptus grandis, Elaeis guineensis, Capsicum annuum, Citrus sinensis, Nicotiana tabacum, yeast, mice, and humans used in this study. Figure S1: ClustalX2 generated alignment of CAF1 protein sequences from A. thaliana, O. sativa, Capsicum annuum, Citrus sinensis, Nicotiana tabacum, yeast (SpCAF1), humans (HsCNOT7), mice, and Zea mays (ZmCAF1). Conserved RNase D-domain residues are marked below the sequence. Figure S2: A list of the motifs. Figure S3: Prediction of the cis-regulatory elements in the promoters. Upstream $1500 \mathrm{bp}$ sequences of each gene promoter were analyzed in the PlantCARE server. Stress-related cis-regulatory elements are spotted in different colors. Table S1: CAF1 gene family in various species. Table S2: List of primers for qRT-PCR analysis in this study. Table S3: Cis-acting elements of PtCAF1 in promoter regions. Table S4: PtCAF1 gene duplication pairs (synteny relationship) in PtCAF1 gene family. Data S1: Analysis data of RNA-Seq and qRT-PCR data. 
Author Contributions: P.W. has written the draft and prepared the manuscript. H.W. and W.S. have designed the experiments. L.L., P.Z., S.Z. and D.L. have reviewed the manuscript and modified. Q.Z. has supervised the manuscript. Q.Z. has funded this project. All authors have read and agreed to the published version of the manuscript.

Funding: This research was supported by the National Natural Science Foundation of China (NSFC) (31570650), and the Priority Academic Program Development of Jiangsu Higher Education Institutions.

Institutional Review Board Statement: Not applicable.

Informed Consent Statement: Not applicable.

Data Availability Statement: Not applicable.

Conflicts of Interest: The authors declare no conflict of interest.

\section{References}

1. Reverdatto, S.V.; Dutko, J.A.; Chekanova, J.A.; Hamilton, D.A.; Belostotsky, D.A. mRNA deadenylation by PARN is essential for embryogenesis in higher plants. RNA 2004, 10, 1200-1214. [CrossRef] [PubMed]

2. Belostotsky, D.A.; Sieburth, L.E. Kill the messenger: mRNA decay and plant development. Curr. Opin. Plant Biol. 2009, 12, 96-102. [CrossRef]

3. Temme, C.; Zaessinger, S.; Meyer, S.; Simonelig, M.; Wahle, E. A complex containing the CCR4 and CAF1 proteins is involved in mRNA deadenylation in Drosophila. EMBO J. 2004, 23, 2862-2871. [CrossRef] [PubMed]

4. Marchese, F.P.; Aubareda, A.; Tudor, C.; Saklatvala, J.; Clark, A.R.; Dean, J.L. MAPKAP kinase 2 blocks tristetraprolin-directed mRNA decay by inhibiting CAF1 deadenylase recruitment. J. Biol. Chem. 2010, 285, 27590-27600. [CrossRef]

5. Meyer, S.; Temme, C.; Wahle, E. Messenger RNA turnover in eukaryotes: Pathways and enzymes. Crit. Rev. Biochem. Mol. Biol. 2004, 39, 197-216. [CrossRef] [PubMed]

6. Chiba, Y.; Green, P.J. mRNA Degradation Machinery in Plants. J. Plant Biol. 2009, 52, 114-124. [CrossRef]

7. Zhao, T.; Huan, Q.; Sun, J.; Liu, C.; Hou, X.; Yu, X.; Silverman, I.M.; Zhang, Y.; Gregory, B.D.; Liu, C.M.; et al. Impact of poly(A)-tail G-content on Arabidopsis PAB binding and their role in enhancing translational efficiency. Genome Biol. 2019, 20, 189. [CrossRef]

8. Chen, X.J.; Zhang, X.H.; Hu, L.D.; Zhang, J.Q.; Jiang, Y.; Yang, Y.; Yan, Y.B. DsCaf1 is involved in environmental stress response of Dunaliella salina. Int. J. Biol. Macromol. 2016, 82, 369-374. [CrossRef]

9. Arae, T.; Morita, K.; Imahori, R.; Suzuki, Y.; Yasuda, S.; Sato, T.; Yamaguchi, J.; Chiba, Y. Identification of Arabidopsis CCR4-NOT Complexes with Pumilio RNA-Binding Proteins, APUM5 and APUM2. Plant Cell Physiol. 2019, 60, 2015-2025. [CrossRef] [PubMed]

10. Tucker, M.; Valencia-Sanchez, M.A.; Staples, R.R.; Chen, J.; Denis, C.L.; Parker, R. The transcription factor associated Ccr4 and Caf1 proteins are components of the major cytoplasmic mRNA deadenylase in Saccharomyces cerevisiae. Cell 2001, 104, 377-386. [CrossRef]

11. Garapaty, S.; Mahajan, M.A.; Samuels, H.H. Components of the CCR4-NOT complex function as nuclear hormone receptor coactivators via association with the NRC-interacting Factor NIF-1. J. Biol. Chem. 2008, 283, 6806-6816. [CrossRef] [PubMed]

12. Zhang, Z.J.; Gao, Q.; Fang, X.D.; Ding, Z.H.; Gao, D.M.; Xu, W.Y.; Cao, Q.; Qiao, J.H.; Yang, Y.Z.; Han, C.; et al. CCR4, a RNA decay factor, is hijacked by a plant cytorhabdovirus phosphoprotein to facilitate virus replication. eLife 2020, 9. [CrossRef]

13. Balu, B.; Maher, S.P.; Pance, A.; Chauhan, C.; Naumov, A.V.; Andrews, R.M.; Ellis, P.D.; Khan, S.M.; Lin, J.W.; Janse, C.J.; et al. CCR4-associated factor 1 coordinates the expression of Plasmodium falciparum egress and invasion proteins. Eukaryot Cell 2011, 10, 1257-1263. [CrossRef]

14. Xu, K.; Bai, Y.; Zhang, A.; Zhang, Q.; Bartlam, M.G. Insights into the structure and architecture of the CCR4-NOT complex. Front. Genet. 2014, 5, 137. [CrossRef] [PubMed]

15. Buschauer, R.; Matsuo, Y.; Sugiyama, T.; Chen, Y.H.; Alhusaini, N.; Sweet, T.; Ikeuchi, K.; Cheng, J.; Matsuki, Y.; Nobuta, R.; et al. The Ccr4-Not complex monitors the translating ribosome for codon optimality. Science 2020, 368, 6488-6912. [CrossRef] [PubMed]

16. Prevot, D.; Morel, A.P.; Voeltzel, T.; Rostan, M.C.; Rimokh, R.; Magaud, J.P.; Corbo, L. Relationships of the antiproliferative proteins BTG1 and BTG2 with CAF1, the human homolog of a component of the yeast CCR4 transcriptional complex: Involvement in estrogen receptor alpha signaling pathway. J. Biol. Chem. 2001, 276, 9640-9648. [CrossRef] [PubMed]

17. Berthet, C.; Morera, A.M.; Asensio, M.J.; Chauvin, M.A.; Morel, A.P.; Dijoud, F.; Magaud, J.P.; Durand, P.; Rouault, J.P. CCR4associated factor CAF1 is an essential factor for spermatogenesis. Mol. Cell Biol. 2004, 24, 5808-5820. [CrossRef]

18. Shi, J.X.; Li, J.S.; Hu, R.; Li, X.M.; Wang, H. CAF1-knockout mice are more susceptive to lipopolysaccharide-induced acute lung injury. J. Inflamm. Res. 2016, 9, 115-121. [CrossRef]

19. Ahuja, I.; de Vos, R.C.; Bones, A.M.; Hall, R.D. Plant molecular stress responses face climate change. Trends Plant Sci. 2010, 15, 664-674. [CrossRef] 
20. Chou, W.L.; Huang, L.F.; Fang, J.C.; Yeh, C.H.; Hong, C.Y.; Wu, S.J.; Lu, C.A. Divergence of the expression and subcellular localization of CCR4-associated factor 1 (CAF1) deadenylase proteins in Oryza sativa. Plant Mol. Biol. 2014, 85, 443-458. [CrossRef]

21. Chou, W.L.; Chung, Y.L.; Fang, J.C.; Lu, C.A. Novel interaction between CCR4 and CAF1 in rice CCR4-NOT deadenylase complex. Plant Mol. Biol. 2017, 93, 79-96. [CrossRef]

22. Hart, K.J.; Oberstaller, J.; Walker, M.P.; Minns, A.M.; Kennedy, M.F.; Padykula, I.; Adams, J.H.; Lindner, S.E. Plasmodium male gametocyte development and transmission are critically regulated by the two putative deadenylases of the CAF1/CCR4/NOT complex. PLoS Pathog. 2019, 15, e1007164. [CrossRef] [PubMed]

23. Walley, J.W.; Kelley, D.R.; Nestorova, G.; Hirschberg, D.L.; Dehesh, K. Arabidopsis deadenylases AtCAF1a and AtCAF1b play overlapping and distinct roles in mediating environmental stress responses. Plant Physiol. 2010, 152, 866-875. [CrossRef] [PubMed]

24. Sarowar, S.; Oh, H.W.; Cho, H.S.; Baek, K.H.; Seong, E.S.; Joung, Y.H.; Choi, G.J.; Lee, S.; Choi, D. Capsicum annuum CCR4associated factor CaCAF1 is necessary for plant development and defence response. Plant J. 2007, 51, 792-802. [CrossRef] [PubMed]

25. Shimo, H.M.; Terassi, C.; Lima Silva, C.C.; Zanella, J.L.; Mercaldi, G.F.; Rocco, S.A.; Benedetti, C.E. Role of the Citrus sinensis RNA deadenylase CsCAF1 in citrus canker resistance. Mol. Plant Pathol. 2019, 20, 1105-1118. [CrossRef]

26. Liang, W.; Li, C.; Liu, F.; Jiang, H.; Li, S.; Sun, J.; Wu, X.; Li, C. The Arabidopsis homologs of CCR4-associated factor 1 show mRNA deadenylation activity and play a role in plant defence responses. Cell Res. 2009, 19, 307-316. [CrossRef]

27. Kwon, T.-M.; Yi, Y.-B.; Nam, J.-S. Overexpression of AtCAF1, CCR4-associated factor 1 homologue in Arabidopsis thaliana, negatively regulates wounding-mediated disease resistance. J. Plant Biotechnol. 2011, 38, 278-284. [CrossRef]

28. Hirayama, T.; Matsuura, T.; Ushiyama, S.; Narusaka, M.; Kurihara, Y.; Yasuda, M.; Ohtani, M.; Seki, M.; Demura, T.; Nakashita, $\mathrm{H}$; et al. A poly(A)-specific ribonuclease directly regulates the poly(A) status of mitochondrial mRNA in Arabidopsis. Nat. Commun. 2013, 4, 2247. [CrossRef] [PubMed]

29. Fang, J.C.; Liu, H.Y.; Tsai, Y.C.; Chou, W.L.; Chang, C.C.; Lu, C.A. A CCR4 Association Factor 1, OsCAF1B, Participates in the alphaAmy3 mRNA Poly(A) Tail Shortening and Plays a Role in Germination and Seedling Growth. Plant Cell Physiol. 2020, 61, 554-564. [CrossRef] [PubMed]

30. Fang, J.C.; Tsai, Y.C.; Chou, W.L.; Liu, H.Y.; Chang, C.C.; Wu, S.J.; Lu, C.A. A CCR4-associated factor 1, OsCAF1B, confers tolerance of low-temperature stress to rice seedlings. Plant Mol. Biol. 2021, 105, 177-192. [CrossRef] [PubMed]

31. Collart, M.A. The Ccr4-Not complex is a key regulator of eukaryotic gene expression. Wiley Interdiscip. Rev. RNA 2016, 7, 438-454. [CrossRef]

32. Parsons, T.J.; Sinkar, V.P.; Stettler, R.F.; Nester, E.W.; Gordon, M.P. Transformation of Poplar by Agrobacterium tumefaciens. Bio/Technol. 1986, 4, 533-536. [CrossRef]

33. Hu, J.; Yang, M.; Lu, M. Advances in biosafety studies on transgenic insect-resistant poplars in China. Biodivers. Sci. 2010, 18, 336-345.

34. Xie, T.; Chen, C.; Li, C.; Liu, J.; Liu, C.; He, Y. Genome-wide investigation of WRKY gene family in pineapple: Evolution and expression profiles during development and stress. BMC Genom. 2018, 19, 490. [CrossRef]

35. Zhu, W.; Guo, Y.; Chen, Y.; Wu, D.; Jiang, L. Genome-wide identification, phylogenetic and expression pattern analysis of GATA family genes in Brassica napus. BMC Plant Biol. 2020, 20, 543. [CrossRef]

36. Movahedi, A.; Zhang, J.; Yin, T.; Qiang, Z. Functional Analysis of Two Orthologous NAC Genes, CarNAC3, and CarNAC6 from Cicer arietinum, Involved in Abiotic Stresses in Poplar. Plant Mol. Biol. Rep. 2015, 33, 1539-1551. [CrossRef]

37. Hui, W.; Movahedi, A.; Xu, C.; Sun, W.; Li, L.; Li, D.; Qiang, Z. Characterization, expression profiling, and functional analysis of a Populus trichocarpa defensin gene and its potential as an anti-Agrobacterium rooting medium additive. Sci. Rep. 2019, 9, 15359-15375. [CrossRef]

38. Wilusz, C.J.; Gao, M.; Jones, C.L.; Wilusz, J.; Peltz, S.W. Poly(A)-binding proteins regulate both mRNA deadenylation and decapping in yeast cytoplasmic extracts. RNA 2001, 7, 1416-1424. [PubMed]

39. Yang, X.; Morita, M.; Wang, H.; Suzuki, T.; Yang, W.; Luo, Y.; Zhao, C.; Yu, Y.; Bartlam, M.; Yamamoto, T.; et al. Crystal structures of human BTG2 and mouse TIS21 involved in suppression of CAF1 deadenylase activity. Nucleic Acids Res. 2008, 36, 6872-6881. [CrossRef] [PubMed]

40. Nishimura, N.; Kitahata, N.; Seki, M.; Narusaka, Y.; Narusaka, M.; Kuromori, T.; Asami, T.; Shinozaki, K.; Hirayama, T. Analysis of ABA hypersensitive germination2 revealed the pivotal functions of PARN in stress response in Arabidopsis. Plant J. 2005, 44, 972-984. [CrossRef]

41. Chong, L.; Guo, P.; Zhu, Y. Mediator Complex: A Pivotal Regulator of ABA Signaling Pathway and Abiotic Stress Response in Plants. Int. J. Mol. Sci 2020, 21, 7755. [CrossRef]

42. Nishimura, N.; Okamoto, M.; Narusaka, M.; Yasuda, M.; Nakashita, H.; Shinozaki, K.; Narusaka, Y.; Hirayama, T. ABA hypersensitive germination2-1 causes the activation of both abscisic acid and salicylic acid responses in Arabidopsis. Plant Cell Physiol. 2009, 50, 2112-2122. [CrossRef] [PubMed]

43. Otsuka, K.; Mamiya, A.; Konishi, M.; Nozaki, M.; Kinoshita, A.; Tamaki, H.; Arita, M.; Saito, M.; Yamamoto, K.; Hachiya, T.; et al. Temperature-dependent fasciation mutants provide a link between mitochondrial RNA processing and lateral root morphogenesis. eLife 2021, 10, 61611-61622. [CrossRef] 
44. Corbin, C.; Drouet, S.; Markulin, L.; Auguin, D.; Laine, E.; Davin, L.B.; Cort, J.R.; Lewis, N.G.; Hano, C. A genome-wide analysis of the flax (Linum usitatissimum L.) dirigent protein family: From gene identification and evolution to differential regulation. Plant Mol. Biol. 2018, 97, 73-101. [CrossRef] [PubMed]

45. Liu, M.; Sun, W.; Ma, Z.; Zheng, T.; Huang, L.; Wu, Q.; Zhao, G.; Tang, Z.; Bu, T.; Li, C.; et al. Genome-wide investigation of the AP2/ERF gene family in tartary buckwheat (Fagopyum Tataricum). BMC Plant Biol. 2019, 19, 84. [CrossRef] [PubMed]

46. Maestrini, P.; Cavallini, A.; Rizzo, M.; Giordani, T.; Bernardi, R.; Durante, M.; Natali, L. Isolation and expression analysis of low temperature-induced genes in white poplar (Populus alba). J. Plant Physiol. 2009, 166, 1544-1556. [CrossRef] [PubMed]

47. Li, W.; Cui, X.; Meng, Z.; Huang, X.; Xie, Q.; Wu, H.; Jin, H.; Zhang, D.; Liang, W. Transcriptional regulation of Arabidopsis MIR168a and argonaute1 homeostasis in abscisic acid and abiotic stress responses. Plant Physiol. 2012, 158, 1279-1292. [CrossRef] [PubMed]

48. Yang, Y.; Lee, J.H.; Poindexter, M.R.; Shao, Y.; Liu, W.; Lenaghan, S.C.; Ahkami, A.H.; Blumwald, E.; Stewart, C.N., Jr. Rational design and testing of abiotic stress-inducible synthetic promoters from poplar cis-regulatory elements. Plant Biotechnol. J. 2021. [CrossRef] [PubMed]

49. Li, S.; Lin, Y.J.; Wang, P.; Zhang, B.; Li, M.; Chen, S.; Shi, R.; Tunlaya-Anukit, S.; Liu, X.; Wang, Z.; et al. The AREB1 Transcription Factor Influences Histone Acetylation to Regulate Drought Responses and Tolerance in Populus trichocarpa. Plant Cell 2019, 31, 663-686. [CrossRef] [PubMed]

50. Li, X.; Gao, S.; Tang, Y.; Li, L.; Zhang, F.; Feng, B.; Fang, Z.; Ma, L.; Zhao, C. Genome-wide identification and evolutionary analyses of bZIP transcription factors in wheat and its relatives and expression profiles of anther development related TabZIP genes. BMC Genom. 2015, 16, 976. [CrossRef]

51. Zhang, H.; Yang, J.; Wang, W.; Li, D.; Hu, X.; Wang, H.; Wei, M.; Liu, Q.; Wang, Z.; Li, C. Genome-wide identification and expression profiling of the copper transporter gene family in Populus trichocarpa. Plant Physiol. Biochem. 2015, 97, 451-460. [CrossRef] [PubMed]

52. Cao, J.; Jiang, M.; Li, P.; Chu, Z. Genome-wide identification and evolutionary analyses of the PP2C gene family with their expression profiling in response to multiple stresses in Brachypodium distachyon. BMC Genom. 2016, 17, 175. [CrossRef]

53. Li, D.; Liu, P.; Yu, J.; Wang, L.; Dossa, K.; Zhang, Y.; Zhou, R.; Wei, X.; Zhang, X. Genome-wide analysis of WRKY gene family in the sesame genome and identification of the WRKY genes involved in responses to abiotic stresses. BMC Plant Biol. 2017, 17, 152. [CrossRef] [PubMed]

54. Faraji, S.; Filiz, E.; Kazemitabar, S.K.; Vannozzi, A.; Palumbo, F.; Barcaccia, G.; Heidari, P. The AP2/ERF Gene Family in Triticum durum: Genome-Wide Identification and Expression Analysis under Drought and Salinity Stresses. Genes 2020, 11, 1464. [CrossRef] [PubMed]

55. Makova, K.D.; Li, W.-H. Divergence in the spatial pattern of gene expression between human duplicate genes. Genome Res. 2003, 13, 1638-1645. [CrossRef]

56. Cannon, S.B.; Mitra, A.; Baumgarten, A.; Young, N.D.; May, G. The roles of segmental and tandem gene duplication in the evolution of large gene families in Arabidopsis thaliana. BMC Plant Biol. 2004, 4, 10. [CrossRef] [PubMed]

57. Li, W.H.; Yang, J.; Gu, X. Expression divergence between duplicate genes. Trends Genet. Tig 2005, 21, 602-607. [CrossRef]

58. Walley, J.W.; Kelley, D.R.; Savchenko, T.; Dehesh, K. Investigating the function of CAF1 deadenylases during plant stress responses. Plant Signal. Behav. 2010, 5, 802-805. [CrossRef] [PubMed]

59. Meinke, D.W. Genome-wide identification of EMBRYO-DEFECTIVE (EMB) genes required for growth and development in Arabidopsis. New Phytol. 2020, 226, 306-325. [CrossRef] [PubMed]

60. Huang, S.-H.; Liu, Y.-X.; Deng, R.; Lei, T.-T.; Tian, A.-J.; Ren, H.-H.; Wang, S.-F.; Wang, X.-F. Genome-wide identification and expression analysis of the GSK gene family in Solanum tuberosum L. under abiotic stress and phytohormone treatments and functional characterization of StSK21 involvement in salt stress. Gene 2021, 766, 145156. [CrossRef] [PubMed]

61. Zhang, H.; Yin, T. Analysis of topology properties in different tissues of poplar based on gene co-expression networks. Tree Genet. Genomes 2019, 16, 178-189. [CrossRef]

62. Jia, H.F.; Chai, Y.M.; Li, C.L.; Lu, D.; Luo, J.J.; Qin, L.; Shen, Y.Y. Abscisic acid plays an important role in the regulation of strawberry fruit ripening. Plant Physiol. 2011, 157, 188-199. [CrossRef] [PubMed]

63. Milborrow, B.V. The Metabolism of Abscisic Acid. J. Exp. Bot. 1970, 21, 17-29. [CrossRef]

64. Joshi-Saha, A.; Valon, C.; Leung, J. Chapter 7-Molecular Mechanisms of Abscisic Acid Action in Plants and Its Potential Applications to Human Health. In Advances in Botanical Research; Turkan, I., Ed.; Academic Press: Cambridge, MA, USA, 2011; Volume 57, pp. 249-292.

65. Nakashima, K.; Yamaguchi-Shinozaki, K. ABA signaling in stress-response and seed development. Plant Cell Rep. 2013, 32, 959-970. [CrossRef] [PubMed]

66. Shinozaki, K.; Yamaguchi-Shinozaki, K. Molecular responses to dehydration and low temperature: Differences and cross-talk between two stress signaling pathways. Curr. Opin. Plant Biol. 2000, 3, 217-223. [CrossRef]

67. Knight, H.; Knight, M.R. Abiotic stress signalling pathways: Specificity and cross-talk. Trends Plant Sci. 2001, 6, 262-267. [CrossRef]

68. Yi, L.; Li, B.; Korpelainen, H.; Yu, F.; Wu, L.; Tong, L.; Liu, M. Mechanisms of drought response in Populus. South. For. J. For. Sci. 2020, 82, 359-366. [CrossRef] 
69. Thakur, A.K.; Kumar, P.; Parmar, N.; Shandil, R.K.; Aggarwal, G.; Gaur, A.; Srivastava, D.K. Achievements and prospects of genetic engineering in poplar: A review. New For. 2021, 2, 361-390. [CrossRef]

70. Yan, C.; Wang, Y.; Lyu, T.; Hu, Z.; Ye, N.; Liu, W.; Li, J.; Yao, X.; Yin, H. Alternative Polyadenylation in response to temperature stress contributes to gene regulation in Populus trichocarpa. BMC Genom. 2021, 22, 53. [CrossRef]

71. Wu, L.; Belasco, J.G. Let Me Count the Ways: Mechanisms of Gene Regulation by miRNAs and siRNAs. Mol. Cell 2008, 29, 1-7. [CrossRef]

72. Wu, L.; Fan, J.; Belasco, J.G. MicroRNAs direct rapid deadenylation of mRNA. Proc. Nat. Acad. Sci. USA 2006, 103, 4034-4039. [CrossRef] [PubMed]

73. Sun, L.; Sun, G.; Shi, C.; Sun, D. Transcriptome analysis reveals new microRNAs-mediated pathway involved in anther development in male sterile wheat. BMC Genom. 2018, 19, 333. [CrossRef] [PubMed]

74. Myburg, A.A.; Grattapaglia, D.; Tuskan, G.A.; Hellsten, U.; Hayes, R.D.; Grimwood, J.; Jenkins, J.; Lindquist, E.; Tice, H.; Bauer, D.; et al. The genome of Eucalyptus grandis. Nature 2014, 510, 356-362. [CrossRef]

75. Tamura, K.; Peterson, D.; Peterson, N.; Stecher, G.; Nei, M.; Kumar, S. MEGA5: Molecular evolutionary genetics analysis using maximum likelihood, evolutionary distance, and maximum parsimony methods. Mol. Biol. Evol. 2011, 28, 2731-2739. [CrossRef] [PubMed]

76. Huang, Z.; Duan, W.; Song, X.; Tang, J.; Wu, P.; Zhang, B.; Hou, X. Retention, Molecular Evolution, and Expression Divergence of the Auxin/Indole Acetic Acid and Auxin Response Factor Gene Families in Brassica Rapa Shed Light on Their Evolution Patterns in Plants. Genome Biol. Evol. 2015, 8, 302-316. [CrossRef] [PubMed]

77. Livak, K.J.; Schmittgen, T.D. Analysis of relative gene expression data using real-time quantitative PCR and the 2(-Delta Delta C(T)) Method. Methods 2001, 25, 402-408. [CrossRef] 